\title{
Galvanomagnetic properties and noise in a barely metallic film of $\mathrm{V}_{2} \mathrm{O}_{3}$
}

\author{
Clara Grygiel* $^{*}$ and Alain Pautrat ${ }^{\dagger}$ \\ Laboratoire CRISMAT, UMR 6508 du CNRS, ENSICAEN et Université de Caen, 6 Bd. Maréchal Juin, 14050 Caen, France \\ Pierre Rodière \\ Institut Néel, CNRS-UJF, BP 166, 38042 Grenoble Cedex 9, France
}

(Received 22 December 2008; revised manuscript received 14 May 2009; published 8 June 2009)

\begin{abstract}
We have measured the magnetotransport properties of a strained metallic $\mathrm{V}_{2} \mathrm{O}_{3}$ thin film. Most of the properties are similar to $\mathrm{V}_{2} \mathrm{O}_{3}$ single crystals that have been submitted to a large pressure. In addition, the resistance noise analysis indicates that conductivity fluctuations are freezing out at $T \approx 10 \mathrm{~K}$. Examination of a range of measurements leads to the conclusion that spins-configuration fluctuations dominate in the lowtemperature regime.
\end{abstract}

DOI: 10.1103/PhysRevB.79.235111

PACS number(s): 73.43.Qt, 73.50.Td

\section{INTRODUCTION}

$\mathrm{V}_{2} \mathrm{O}_{3}$ single crystals undergo a first-order metal-insulator (MI) transition at $T \approx 160 \mathrm{~K}$ with antiferromagnetic ordering of vanadium spins associated to a structural transition. ${ }^{1}$ In vanadium deficient samples, the metallic phase was shown to be stabilized down to $T \approx 10 \mathrm{~K},{ }^{2}$ where a spin-density wave condenses as indicated by a clear increase in the resistivity. ${ }^{3}$ At roughly the same temperature, the Hall resistance shows a maximum, which was first attributed to the skew scattering in an ordered magnetic state. ${ }^{4}$ Stoichiometric $\mathrm{V}_{2} \mathrm{O}_{3}$ behaves differently. When submitted to a high hydrostatic pressure $P \geq 26$ kbar, its longitudinal resistivity decreases monotonically with a decrease in temperature and any gap opening can be observed. This indicates a fully suppressed metal to the insulator transition down to the lowest temperature without any trace of magnetic ordering. ${ }^{2}$ On the other hand, the Hall resistance still shows a maximum at $T \approx 10 \mathrm{~K},{ }^{5}$ which may be explained by dominant spin fluctuations ${ }^{6}$ or strong electronic correlations ${ }^{7}$ in the low-temperature range. It has been reported that the spin susceptibility of metallic $\mathrm{V}_{2} \mathrm{O}_{3}$ under pressure exhibits also an anomaly at $T \approx 10 \mathrm{~K}$ which has been associated with either low-energy magnetic excitations or a kind of pseudogap formation. ${ }^{8}$ There is a need for complementary experiments to clarify the origin of this anomaly.

When $\mathrm{V}_{2} \mathrm{O}_{3}$ is epitaxially grown over a substrate, clamping of the film can prevent the structural transition. ${ }^{9}$ As a consequence, a metallic state which mimics the highly pressured state is observed. Macroscopically, a distribution of strains in the thin film can lead to phase coexistence. The resistivity exhibits thermal hysteresis due to metastable states and is a nonergodic quantity in parts of the phase diagram, e.g., the quantitative analysis of the measured values is ambiguous. ${ }^{10}$ However, using a microbridge as a local probe, it is possible to isolate a pure metallic state without any thermal hysteresis and no trace of the MI transition. In this paper, we discuss the transport properties of a strained $\mathrm{V}_{2} \mathrm{O}_{3}$ thin film in the so-called barely metallic side. We have performed conventional galvanomagnetic measurements and noise measurements. We will show that most of the properties are comparable to those observed in single crystals which were submitted to high pressure. The small size of the system makes the statistical averaging less effective and, thus, makes the extraction of information from noise measurements possible. As we will discuss below, the analysis of conductivity fluctuations provide different insights into the low-temperature ground state of metallic $\mathrm{V}_{2} \mathrm{O}_{3}$.

\section{EXPERIMENTAL}

The $\mathrm{V}_{2} \mathrm{O}_{3}$ thin film was grown on a substrate of (0001)oriented sapphire using the pulsed laser deposition from a $\mathrm{V}_{2} \mathrm{O}_{5}$ target. The details of the growth conditions and some structural and microstructural characterization have been reported previously. ${ }^{9}$ In particular, the evolution of unit-cell parameters was shown to be inconsistent with oxygen nonstoichiometry. ${ }^{9}$ The film studied here is a 230 - $\AA$-thick sample, with a low rms roughness of $0.47 \mathrm{~nm}$ (averaged over $3 \times 3 \mu \mathrm{m}^{2}$ ). It was patterned using UV photolithography and argon-ion etching to form a bridge of length $\times$ width $=200$ $\times 100 \mu \mathrm{m}^{2}$. Silver contact pads were connected using aluminum-silicon wires (or copper wires for measurements at temperature below $2 \mathrm{~K}$ ) which were attached by the ultrasonic bonding for four-probe and Hall-effect measurements. Measurements for $T \leq 2 \mathrm{~K}$ were performed in a He-3 cryostat. Transport measurements were taken at temperatures between 2 and $400 \mathrm{~K}$ in a physical properties measurement system (PPMS) from Quantum Design. All magnetotransport measurements have been performed with the magnetic field along the $c$ axis, i.e., perpendicular to the film. For noise measurements, we used a home-made sample holder and external electronics to acquire the resistance-time series and spectrum noise (the acquisition part consists of homeassembled low noise preamplifiers, a spectrum analyzer SR760 and a dynamic signal analyzer NI-4551). The final resolution is dominated by the low noise current supply (current noise: $0.2 \mathrm{nA} / \mathrm{Hz}^{1 / 2}$ ) which leads to an equivalent noise of $100 \mathrm{nV} / \mathrm{Hz}^{1 / 2}$ when the sample with $R \approx 0.5 \mathrm{k} \Omega$ is biased at low temperature. This is higher than both the equivalent Johnson thermal noise and the preamplifier noise. The length dependence of the noise measured between different arms of the bridge allows to conclude that contact noise is not important here. ${ }^{11}$ 


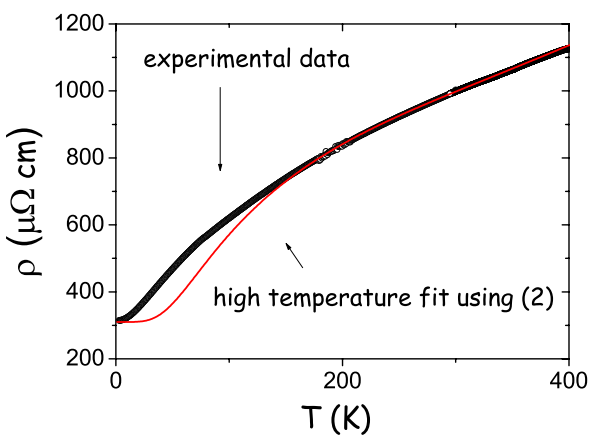

FIG. 1. (Color online) Resistivity of the metallic $\mathrm{V}_{2} \mathrm{O}_{3}$ microbridge as function of temperature. Also shown is a fit of the hightemperature part with the formula $(2)\left(\theta=300 \mathrm{~K}, \rho_{\text {sat }}=1 \mathrm{~m} \Omega \mathrm{cm}\right)$.

\section{RESULTS}

\section{A. Magnetotransport properties}

Figure 1 shows the resistivity of the metallic microbridge as a function of the temperature. The resistivity is thermally reversible and Ohmic for applied currents up to at least $1 \mathrm{~mA}$ and from $T=400$ down to $2 \mathrm{~K}$. Both the functional form of the resistivity and the absolute value were close to what is measured in crystals under high pressure. ${ }^{2}$ In particular, the resistivity tends to curve downward at high temperature but takes a value above the three-dimensional Ioffe-Regel limit for metallic conductivity. This is often observed in so-called bad metals, and a large $\rho_{\text {sat }} \approx 1 \mathrm{~m} \Omega \mathrm{cm}$ has been discussed as a possible consequence of interacting electrons. ${ }^{12}$ For a correlated metal, a lot of different terms can play a role in electronic scattering; but at high temperature a major contribution from phonons can be still expected. The electronphonon contribution to the resistivity is usually described by the Bloch-Grüneisen formula,

$$
\rho_{\mathrm{ph}}=c T^{5} / \theta 6 \int_{0}^{\theta / T} x^{5}\left[\left(e^{x}-1\right)\left(1-e^{-x}\right)\right]^{-1} d x,
$$

where $\theta$ is the Debye temperature and $c$ is a constant, describing the electron-phonon interaction. The hightemperature data were adjusted to the phenomenological parallel resistor with a saturation resistivity acting as a shunt, ${ }^{13}$

$$
\rho^{-1}=\rho_{\text {sat }}^{-1}+\left(\rho_{0}+\rho_{\mathrm{ph}}\right)^{-1} .
$$

A good fit is obtained for the temperature range $130 \mathrm{~K}$ $\leq T \leq 400 \mathrm{~K}$. We found an approximative residual resistivity $\rho_{0} \approx 320 \mu \Omega \mathrm{cm}$ which will be refined below, a saturation resistivity $\rho_{\text {sat }} \approx 1 \mathrm{~m} \Omega \mathrm{cm}$, and a Debye temperature $\theta$ $=300 \mathrm{~K}$. The deduced Debye temperature was close to half the value of bulk samples $\left(\theta_{\text {bulk }} \approx 560 \mathrm{~K}\right)$, which could be qualitatively explained by a size effect ${ }^{14}$ and/or by the stress in the film. For $T \leq 140-150 \mathrm{~K}$, an additional contribution is needed to describe the resistivity. In particular, at the lowest temperatures, where the electron-phonon scattering was clearly negligible $(T \leq 20 \mathrm{~K})$, a quadratic temperature dependence of the resistivity was observed in the form of $\rho_{T^{2}}$ $=A T^{2}+\rho_{0}$, with $A=0.073 \mu \Omega \mathrm{cm} \mathrm{K}^{-2}$ and a more precise value of the residual resistivity $\rho_{0} \approx 316 \mu \Omega \mathrm{cm}$ (Fig. 2). This Fermi-liquid-like dependence has been reported for

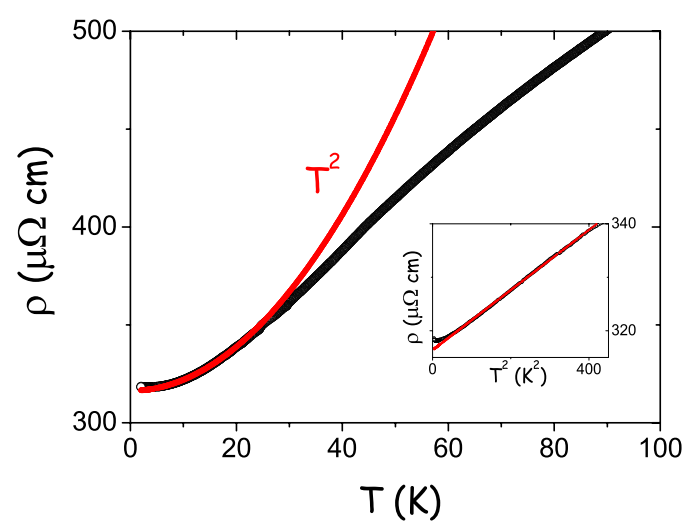

FIG. 2. (Color online) Resistivity of the metallic $\mathrm{V}_{2} \mathrm{O}_{3}$ microbridge as a function of temperature for $T \leq 100 \mathrm{~K}$ and $T^{2}$ fit of the low-temperature part. Shown in the inset is a zoom of $\rho$ as function of $T^{2}$. The temperature variation in the resistivity is quadratic for $10 \mathrm{~K} \leq T \leq 20 \mathrm{~K}$.

single crystals of $\mathrm{V}_{2} \mathrm{O}_{3}$ submitted to a high pressure ${ }^{15,16}$ and, more recently, in strained thin films. ${ }^{17}$ This dependence is typical of electron-electron scattering ${ }^{18,19}$ which is the general interpretation in $\mathrm{V}_{2} \mathrm{O}_{3}$. Spin fluctuations can also lead to a quadratic temperature dependence at low temperature. ${ }^{6} \mathrm{We}$ note also that the functional form of $\rho(T)$ over the full temperature range was not very different from what was expected taken in spin-fluctuations theories. ${ }^{6,20}$ The $T^{2}$ variation in the resistivity did not extend down to the lowest temperature, as can be seen in Fig. 2. Here, an excess of resistivity was observed when $T \leq 10 \mathrm{~K}$. After carefully subtracting $\rho_{T^{2}}$ from the measured resistivity at low temperature $(T \leq 20 \mathrm{~K})$, a logarithmic dependence of the resistivity was observed from $T \leq 10 \mathrm{~K}$ down to $2 \mathrm{~K}$ (Fig. 3). This is the limiting temperature in our PPMS cryostat. Measurements in another film of the same thickness at the lowest temperature indicated that this logarithmic dependence extends down to $400 \mathrm{mK}$. Kondo effect (scattering by magnetic impurities) or weak localization corrections can be made to explain that the scattering increases at low temperatures with such characteristics. The $T^{1 / 2}$-dependent conductivity which is characteris-

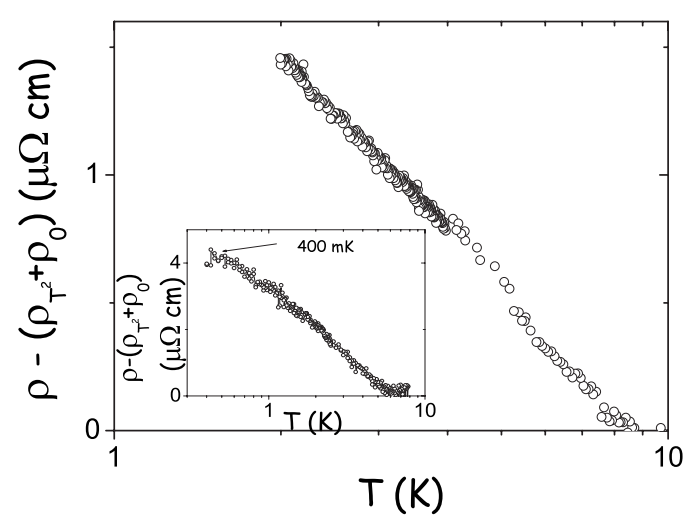

FIG. 3. Variation in the resistivity corrected from the quadratic temperature dependence and from the residual resistance. A logarithm dependence can be observed for $2 \mathrm{~K} \leq T \leq 10 \mathrm{~K}$. Shown in the inset are the same characteristics in a film of the same thickness and measured down to the lowest temperature $(400 \mathrm{mK})$. 


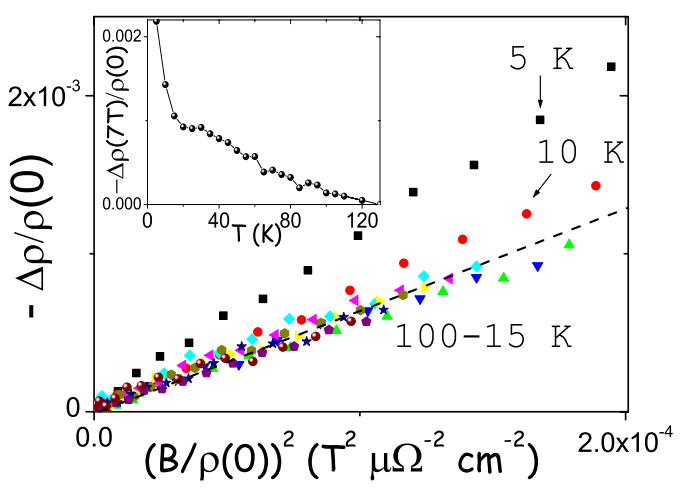

FIG. 4. (Color online) A Kohler's plot of the magnetoresistance $\Delta \rho(B) / \rho(0)$ as a function of $[B / \rho(0)]^{2}$ for temperature from 100 to $5 \mathrm{~K}$. The dashed line is guide for the eyes. Shown in the inset is the magnetoresistance at $7 \mathrm{~T}$ as a function of the temperature.

tic of electron-electron scattering in a disordered medium has been proposed as an explanation for the low-temperature upturn in pressurized $\mathrm{V}_{2} \mathrm{O}_{3}$ resistivity. ${ }^{2,16}$ This, however, does not provide a satisfactory fit for our sample.

In addition to the excess of resistivity, a negative longitudinal magnetoresistance (MR) appears for $T \leq 130 \mathrm{~K}$ (inset of Fig. 4). Its magnetic field dependence is quadratic from $B=0$ to $7 \mathrm{~T}$ and for all temperatures down to $2 \mathrm{~K}$. The origin of the quadratic dependence of a negative magnetoresistance is uncertain. $^{21}$ One possible mechanism is the twodimensional weak localization as far as $B \ll \hbar /\left(4 e L_{\varphi}^{2}\right)$, where $L_{\varphi}$ is the coherence length. ${ }^{22}$ Quadratic negative magnetoresistance is also a common feature of Kondo-like magnetic metals, where the magnetic field tends to suppress the spin fluctuations. Remarkably, the absolute value of the MR shows a notable increase for $T \leq 10 \mathrm{~K}$, clearly evidenced by tracing the Kohler's plot. The idea behind Kohler's rule is that in conventional isotropic metals, the magnitude of MR is fixed by a single-scattering time $\tau(T) \propto 1 / \rho(T)$ and implies that $\Delta \rho(B) / \rho(0)=F(B \tau)$, where $F$ is a function dependent on the details of the electronic structure. Due to its quite large generality, the Kohler's rule is expected to apply in a Fermi liquid also and has been used as a probe of non-Fermi-liquid ground states. In the limit of the carrier density being temperature independent, ${ }^{23}$ Kohler's rule written in its simplest form is $\Delta \rho(B) / \rho(0)=F[B / \rho(0)]$. In Fig. 4, it can be seen that $\Delta \rho(B) / \rho(0)$ plotted as a function of $[B / \rho(0)]^{2}$ for different temperatures gives a single curve, i.e., Kohler's rule is fulfilled when $T \geq 10 \mathrm{~K}$. The strong increase in the MR below $10 \mathrm{~K}$ was thus associated with a departure from Kohler's rule. The Hall resistance also presented a maximum in the 10-20 K range (Fig. 5), as previously reported both for single crystals and for thin films. ${ }^{4,5,17}$ Since it was observed in the absence of a clear magnetic transition, a dominant effect of spin fluctuation has been proposed. ${ }^{5,6}$ Such a maximum can also be expected from strong correlation effects. ${ }^{7}$ In summary of this part, we have confirmed that the magnetotransport properties of metallic $\mathrm{V}_{2} \mathrm{O}_{3}$ thin film present similarities with $\mathrm{V}_{2} \mathrm{O}_{3}$ crystals under pressure such as the $T^{2}$ variation in the resistivity and the maximum of the Hall effect at $T \approx 10 \mathrm{~K}$. In addition, we have shown that the $10 \mathrm{~K}$ anomaly reflects also in the MR and in a Kondo-like increase

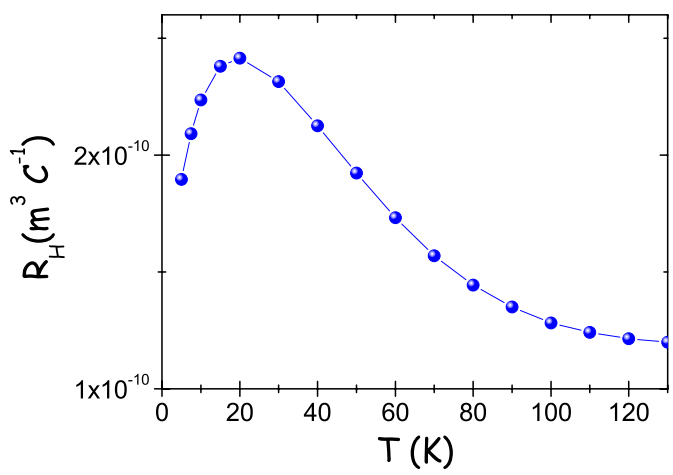

FIG. 5. (Color online) Hall resistance as a function of the temperature, showing the maximum at low temperature.

in the resistivity at low temperature. Both electronic correlations and spin fluctuations can be used as qualitative explanations.

\section{B. Noise measurements as a complementary tool}

Benefiting from the small size of our sample, we have studied conductivity fluctuations by measuring the resistance noise. We have measured a set of resistance-time series at various temperatures. The temperature was allowed to stabilize during sample cooling before each measurement series. The power spectral density (or noise spectrum) $S_{\mathrm{RR}}(f)$ is then calculated. In ordinary metals, the electronic noise generally comes from the motion of atomic impurities or defects and the temperature dependence is dependent by the underlying mechanisms, e.g., thermal activation or eventually tunneling at low temperature. At high temperature $T>100 \mathrm{~K}$, we have observed resistance switches insensitive to magnetic field during long periods, which recall the hydrogen hopping noise common in several metals. ${ }^{24}$ The fractional change in resistance was between $2 \times 10^{-5}-10^{-4}$, which is large for individual hopping and implied a collective motion. Note that this switching noise strongly evolved over a long period, as it was not observed 1 month after the first measurements, showing that the involved defects were mostly nonequilibrium defects left in the film after deposition. In addition, reproducible $1 / f$ noise can be observed and this noise will be discussed now.

Shown in Fig. 6 is typical noise spectra measured on our film. They are very close to $1 / f$ over the whole temperature range. The temperature dependence of this normalized noise exhibits a nonmonotonic variation (Fig. 7), with a plateau in the range $10 \mathrm{~K}<T<20 \mathrm{~K}$. The sudden decrease in the noise at $T \approx 10 \mathrm{~K}$ is remarkable. To give 1 order of magnitude of the noise level, we use the phenomenological Hooge parameter which is, for $1 / f$ noise, $\gamma=\left[S_{\mathrm{RR}}(f) f\right] / R^{2} n_{c} V$ where $n_{c}$ is the carriers density and $V$ is the (probed) sample volume. ${ }^{25}$ For homogeneous and pure metals, typical values are in the range $\gamma \approx 10^{-2}-10^{-3}$. Taking $n_{e}=5 \times 10^{22} \mathrm{~cm}^{-3}$ deduced from our Hall-effect measurements, we found $\gamma$ $\approx 10^{-1}$ at room temperature, which is large, but not unusually large for a metalliclike thin film and is consistent with a moderately higher concentration of defects in films than in bulk. Noise in metals can be usually explained with the 


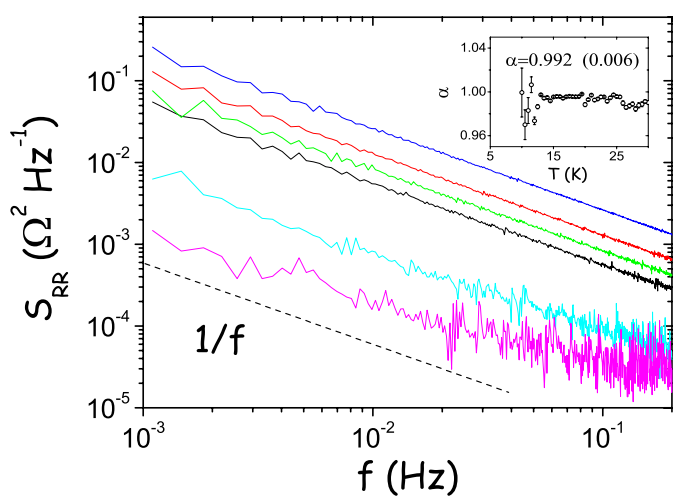

FIG. 6. (Color online) $1 / f^{\alpha}$ noise spectra obtained from the resistance-time series at $T=5,10,30,25,20$, and $15 \mathrm{~K}$ from the bottom to the top. Shown in the inset is the value of the exponent $\alpha$ as a function of the temperature.

Duttah-Dimon-Horn (DDH) analysis, which assumes that the electronic fluctuations come from smeared kinetics of defects with a distribution of activation energies. ${ }^{26}$ When the defect relaxation is activated and typical energy is in the $\mathrm{eV}$ range, the noise generally increases with temperature. However, there is at least one report of a noise maximum at low temperature which, however, follows the DDH framework. It has been interpreted as evidence of low-energy excitations in an oxide. ${ }^{27}$ In the DDH modeling, there is a direct relation between the noise spectral exponent $\alpha$ and the temperature dependence of the noise in the form $\alpha(T)=1$ $-\left(\partial \ln S_{\mathrm{RR}} / \partial \ln T-1\right) / \ln \left(2 \pi f \tau_{0}\right)$, where $\tau_{0}^{-1}$ is the attempt frequency. We have checked this relation and found that unrealistically large $\tau_{0}$ values are needed to approach the very small variation in $\alpha(T)$. In parallel, the temperature of the noise maximum/plateau does not show any frequency dependence. Actually, pure $1 / f$ noise should follow $S_{\mathrm{RR}} \propto T$ in the DDH model, as we observed at high temperature (Fig. 7), and a maximum is not expected. We conclude that the lowtemperature noise maximum does not come from thermally activated excitations. It can neither be explained by temperature fluctuations which would lead to a too weak temperature dependence of the noise. In disordered metals at low temperature, universal conductance fluctuations (UCFs) can in principle take place. ${ }^{28}$ In the UCF model, the noise grows at low temperature due to the enhancement of the effective coupling between disorder configurations and resistance changes. UCF can explain why $S_{\mathrm{RR}}$ rises when the temperature decreases but not the apparent freezing of conductivity fluctuations for $T \leq 10 \mathrm{~K}$. Since we observe a decrease in the resistance noise when a magnetic field is applied in the $10-30 \mathrm{~K}$ range (a factor of 0.6 at $B=7 \mathrm{~T}$ and at $T=15 \mathrm{~K}$, see the inset of Fig. 7), a spin-coupling origin of this noise

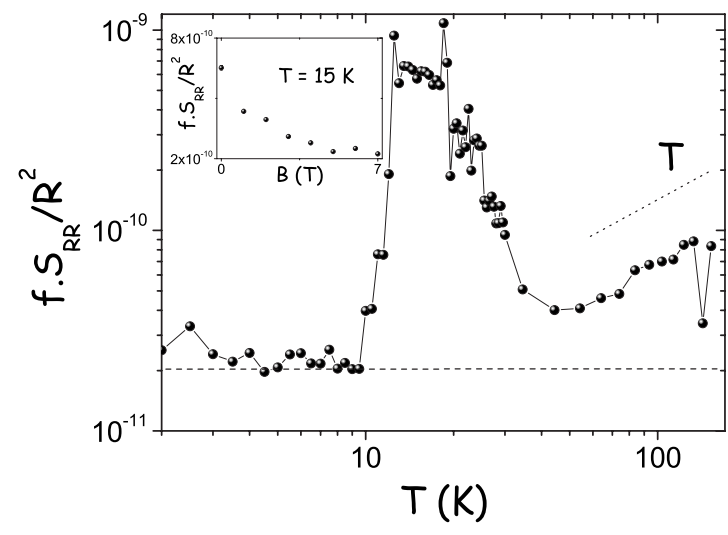

FIG. 7. Normalized resistance noise as a function of the logarithm of the temperature $(f=0.1 \mathrm{~Hz})$. Note the sharp decrease at $T \approx 10 \mathrm{~K}$. The horizontal dotted line corresponds to the resolution of the measurement. Shown in the inset is the normalized noise as a function of the magnetic field for $T=15 \mathrm{~K}(f=0.1 \mathrm{~Hz})$.

can be suggested. The blocking of magnetic fluctuations at low temperature with non-Arrhenius slowing down makes us think of spin glasses. ${ }^{29}$ Note, however, that some spin glasses do not show a decrease but a growing of noise at low temperature due to the UCF coupling. ${ }^{30}$ To probe, directly, spins freezing with resistance noise, the sensitivity of the resistance to spins should be independent of the temperature and the coupling should be local. ${ }^{30}$ This appears to be the case with our sample. Note that in some intermetallic compounds, similar properties, such as a quadratic temperature dependence of the resistivity close to a peak in the Hall coefficient, have been interpreted as a spin-glass type of freezing at low temperature. ${ }^{31,32} \mathrm{~A}$ precise determination of the magnetic disorder is required to better understand its role in the lowtemperature galvanomagnetic properties of $\mathrm{V}_{2} \mathrm{O}_{3}$.

To conclude, we have measured a large array of transport properties in a $\mathrm{V}_{2} \mathrm{O}_{3}$ film in the barely metallic side. We found strong similarities with properties of single crystals submitted to a high pressure and some different features such as a low-temperature noise maximum coupled to anomalous magnetotransport properties. A central role of spinconfiguration fluctuations, which freeze at low temperature, has been proposed. Future experiments directly sensitive to magnetic order would be extremely complementary. Noise experiments on single crystals, if feasible, would be-alsovery interesting to compare with our measurements in films.

\section{ACKNOWLEDGMENTS}

A.P. thanks W. Prellier for comments on the paper and C.G. thanks S. McMitchell. 
*Present address: Department of Chemistry, The University of Liverpool, Liverpool L69 7ZD, United Kingdom.

†alain.pautrat@ensicaen.fr

${ }^{1}$ D. B. Mcwhan, T. M. Rice, and J. P. Remeika, Phys. Rev. Lett. 23, 1384 (1969).

${ }^{2}$ S. A. Carter, T. F. Rosenbaum, J. M. Honig, and J. Spalek, Phys. Rev. Lett. 67, 3440 (1991).

${ }^{3}$ W. Bao, C. Broholm, S. A. Carter, T. F. Rosenbaum, G. Aeppli, S. F. Trevino, P. Metcalf, J. M. Honig, and J. Spalek, Phys. Rev. Lett. 71, 766 (1993).

${ }^{4}$ T. F. Rosenbaum, A. Husmann, S. A. Carter, and J. M. Honig, Phys. Rev. B 57, R13997 (1998).

${ }^{5}$ S. Klimm, M. Herz, R. Horny, G. Obermeier, M. Klemm, and S. Horn, J. Magn. Magn. Mater. 226-230, 216 (2001).

${ }^{6}$ O. Narikiyo and K. Miyake, J. Phys. Soc. Jpn. 64, 2730 (1995).

${ }^{7}$ J. Merino and R. H. McKenzie, Phys. Rev. B 61, 7996 (2000).

${ }^{8}$ M. Takigawa, E. T. Ahrens, and Y. Ueda, Phys. Rev. Lett. 76, 283 (1996).

${ }^{9}$ C. Grygiel, A. Pautrat, W. Prellier, B. Mercey, W. C. Sheets, and L. Mechin, J. Phys.: Condens. Matter 20, 472205 (2008).

${ }^{10}$ C. Grygiel, A. Pautrat, W. Prellier, and B. Mercey, EPL 84, 47003 (2008).

${ }^{11}$ A. C. Marley, M. B. Weissman, R. L. Jacobsen, and G. Mozurkewich, Phys. Rev. B 44, 8353 (1991).

${ }^{12}$ O. Gunnarsson, M. Calandra, and J. E. Han, Rev. Mod. Phys. 75, 1085 (2003).

${ }^{13}$ H. Wiesmann, M. Gurvitch, H. Lutz, A. Ghosh, B. Schwartz, M. Strongin, P. B. Allen, and J. W. Halley, Phys. Rev. Lett. 38, 782 (1977).

${ }^{14}$ G. Kästle, H.-G. Boyen, A. Schröder, A. Plettl, and P. Ziemann, Phys. Rev. B 70, 165414 (2004).

${ }^{15}$ S. A. Carter, T. F. Rosenbaum, M. Lu, H. M. Jaeger, P. Metcalf, J. M. Honig, and J. Spalek, Phys. Rev. B 49, 7898 (1994).
${ }^{16}$ S. Mederle-Hoffmeister, S. Klimm, M. Klemm, and S. Horn, Physica B 259-261, 851 (1999).

${ }^{17}$ C. Grygiel, Ch. Simon, B. Mercey, W. Prellier, R. Frésard, and P. Limelette, Appl. Phys. Lett. 91, 262103 (2007).

${ }^{18}$ W. G. Baber, Proc. R. Soc. London, Ser. A 158, 383 (1937).

${ }^{19}$ W. E. Lawrence and J. W. Wilkins, Phys. Rev. B 7, 2317 (1973).

${ }^{20}$ R. Jullien, M. T. Béal-Monod, and B. Coqblin, Phys. Rev. B 9, 1441 (1974).

${ }^{21}$ J. N. Huiberts, R. Griessen, R. J. Wijngaarden, M. Kremers, and C. Van Haesendonck, Phys. Rev. Lett. 79, 3724 (1997).

${ }^{22}$ We deduce a higher bound $L_{\varphi} \leq 5 \mathrm{~nm}$. This low value of the coherence length could be attributed to some defects due to the strains in the film at low temperature rather than to a low carriers density $n_{e}$ because Hall-effect measurements give $n_{e} \approx 4$ $\times 10^{22} / \mathrm{cm}^{3}$ at room temperature.

${ }^{23}$ N. Luo and G. H. Miley, Physica C 371, 259 (2002).

${ }^{24}$ R. P. Michel and M. B. Weissman, Phys. Rev. B 50, 15796 (1994).

${ }^{25}$ R. D. Black, P. J. Restle, and M. B. Weissman, Phys. Rev. B 28, 1935 (1983).

${ }^{26}$ P. Dutta, P. Dimon, and P. M. Horn, Phys. Rev. Lett. 43, 646 (1979); P. Dutta and P. M. Horn, Rev. Mod. Phys. 53, 497 (1981).

${ }^{27}$ A. Ghosh, A. K. Raychaudhuri, R. Sreekala, M. Rajeswari, and T. Venkatesan, Phys. Rev. B 58, R14665 (1998).

${ }^{28}$ S. Feng, P. A. Lee, and A. D. Stone, Phys. Rev. Lett. 56, 1960 (1986).

${ }^{29}$ R. P. Michel and M. B. Weissman, Phys. Rev. B 47, 574 (1993).

${ }^{30}$ N. E. Israeloff, M. B. Weissman, G. J. Nieuwenhuys, and J. Kosiorowska, Phys. Rev. Lett. 63, 794 (1989).

${ }^{31}$ N. Ali, W. R. Datars, G. Kozlowski, and S. B. Woods, J. Phys. F: Met. Phys. 17, 143 (1987).

${ }^{32}$ S. P. McAlister, J. Appl. Phys. 49, 1616 (1978). 